\title{
Maternal collapse and perimortem caesarean section
}

\author{
Alison McDougall', Hasthika Ellepola ${ }^{23}$, Sam Seneviratna', Mano Haran ${ }^{4}$ \\ Sri Lanka Journal of Obstetrics and Gynaecology 2012; 34: 165-167
}

\section{Introduction}

Maternal collapse is a rare occurrence but understanding potential causes and initiating prompt management can aid to reduce adverse outcomes. Causes of maternal collapse, with a mnemonic, can be seen in Table $1^{1}$. Maternal collapse is any event that affects the cardiorespiratory or brain that results in reduced or absent consciousness. A collapse may be as simple as a vasovagal episode or it may be a cardiac event with unfavorable outcomes. It may occur at any stage in pregnancy until six weeks post partum and may, despite all efforts, result in the death of the mother and baby ${ }^{2}$. It is difficult to determine an accurate rate of maternal collapse, but it is estimated that the true rate of maternal collapse lies somewhere between 14 and 600 per 100,000 births ${ }^{2}$. As doctors, we know of many clinical signs that may precede, the collapse of a patient, however, as this case demonstrates, there may be little warning.

\section{Table 1. Causes of maternal collapse ${ }^{1}$}

\section{BEAU-CHOPS}

Bleeding, disseminated intravascular coagulation

Embolism: coronary, pulmonary, amniotic fluid embolism

Anaesthetic complications

Uterine atony

Cardiac disease: myocardial ischaemia, aortic dissection, cardiomyopathy

Hypertension, preeclampsia, ecclampsia

Other differential diagnoses not related to pregnancy Plancental abruption, placenta previa

Sepsis

\footnotetext{
${ }^{1}$ Registrar, ${ }^{2}$ Consultant, Department of Obstetrics and Gynaecology, Logan Hospital, Meadowbrook, Queensland 4131, Australia.

3 Senior Lecturer, School of Medicine, University of Griffith, Queensland, Australia.

${ }^{4}$ Associate Professor, School of Medicine, University of Griffith and Director Logan Hospital, Queensland, Australia.

Correspondence: Hasthika Ellepola

E-mail: Hasthika_Ellepola@health.qld.gov.au
}

\section{Case report}

A 35 year old woman, gravida 3 parity 2, was admitted to a busy suburban hospital for a routine induction of labour for prolonged pregnancy at 40+10. She had a two previous uncomplicated pregnancies resulting in normal vaginal deliveries. This pregnancy was also deemed low risk and had been without complication. As her cervix was unfavorable, her induction of labour was commenced with $1 \mathrm{mg}$ of prostaglandin gel, which was given at 1900 hours. The patient remained on the ward overnight and was transferred to the Birth Suite for review at 0600 the following day. At this examination, her cervix was three centimeters dilated and an artificial rupture of the membranes was performed, intravenous syntocin was commenced as per protocol at 0715 and continuous external fetal monitoring was initiated. She began contracting regularly at 0930 and continued on $24 \mathrm{mls} /$ hour of syntocin. There were no concerns voiced by the patient, her partner or the midwife caring for the patient.

At approximately 1205, the midwife caring for the patient contacted the team leader in response to a fetal bradycardia. As the team leader entered the room, the patient became unresponsive. The emergency buzzer was activated and 3 obstetric consultants and 2 obstetric registrars promptly arrived. The labouring patient was unresponsive, there was not a recordable pulse and the cardiotocograph revealed an ongoing fetal bradycardia with the heart rate recorded at 70 beats per minute. Resuscitation was commenced and a code blue was called.

After approximately 4 minutes of unsuccessful cardiopulmonary resuscitation, the team, which now consisted of multiple specialty senior obstetric consultants, registrars, as well as ICU and anaesthetic consultants, agreed to perform a perimortem caesarean section in the Birth Suite.

While resuscitation efforts continued, a lower abdominal transverse incision was made, followed by a lower uterine transverse incision and an asphyxiated female infant was delivered and handed to the paediatric team. This occurred in less that 1 minute after the decision for the caesarean section occurred. The neonates Apgar scores were 1 at 1 minute, 5 at 5 minutes. Cord blood gases were not collected. 
Maternal resuscitation continued and the presence of disseminated intravascular coagulation shortly became apparent. Despite all resources and team efforts, the patient did not regain consciousness and died in ICU. For cultural reasons, the family declined a postmortem examination. It is likely the patient suffered from an amniotic fluid embolism but a differential diagnosis includes a massive thromboembolism.

The availability of three specialists in three specialty, the quick response of the team of registrars and midwives saved the baby and a very well coordinated effort was applauded by the state mortality review committee.

\section{Discussion}

According to the latest figures from the Australian Institute of Health and Welfare, there were 65 maternal deaths in Australia from 1st January 2003 to 31st December $2005^{3}$. Also from this report, the leading causes of direct and indirect maternal deaths can be seen in Table 2. Direct deaths are deaths from obstetric complications, for example post partum haemorrahge, while indirect deaths are deaths from existing disease, which may be exacerbated by pregnancy.

Table 2. Cause of maternal death, Australia 2003-2005

\begin{tabular}{ll}
\hline Cause of death $\quad$ No. of deaths \\
\hline
\end{tabular}

\section{Direct}

Amniotic fluid embolism

Hypertensive disorders

Thrombosis and thromboembolism

Obstetric haemorrhage

Cardiac conditions

Infections

Deaths associated with anaesthesia

Non-genital tract haemorrhage

Thrombotic thrombocytopaenia purpura

\section{Indirect}

Cardiac conditions

Psychiatric causes

Non-obstetric haemorrhage

Infection

Hypertension

Other

Total
Resuscitation of the pregnant patient can be difficult. From 20 weeks gestation, the gravid uterus can place pressure on the aorta and inferior vena cava, thus decreasing venous return and reducing stroke volume and cardiac output ${ }^{4}$. The Royal College of Obstetricians and Gynaecologists (RCOG) advise that a left lateral tilt of 15 degrees on a firm surface will relieve aortal and vena caval compression in the majority of patients and will still allow effective chest compressions to be performed ${ }^{2}$. The American Heart Association suggests manually displacing the uterus while the patient is in the supine position to relieve aortocaval compression and to allow for effect chest compressions during CPR ${ }^{1}$. Previously, there has been concern regarding the defibrillation of pregnant patients. While there is a small risk of inducing foetal arrhythmias, defibrillation at standard advanced cardiac life support settings should be carried out if required $^{1,2}$. Fetal monitors should be removed prior to defibrillation.

The decision to perform a perimortem caesarean section is a difficult one and timing of the operation is crucial for both foetal and maternal survival. In 1986, Katz et al. suggested the Four Minute Rule: "Caesarean delivery should be begun within four minutes and the infant delivered within five minutes after maternal cardiac arrest" ${ }^{\prime \prime}$. In 2005, Katz reviewed 38 case reports of perimortem caesearn section for delivery of singletons, twins or triplets, between 1986 and 2004 . They found that in 30 of the 38 perimortem caesarean sections, there were surviving infants. Of the 38 mothers, only 16 survived as 14 were though to be unresuscitatable and for the other 8 deaths, the cardiac arrests were from lethal insults ${ }^{6}$. The conclusion from this review supports performing a perimortem caesarean section within four minutes if resuscitation is ineffective ${ }^{6}$.

Maternal collapse leading to maternal death is a rare event in the developed world. The World Health Organization (WHO) defines maternal death as, "The death of a woman while pregnant or within 42 days of termination of pregnancy, irrespective of the duration and site of the pregnancy, from any cause related to or aggravated by the pregnancy or its management but not from accidental or incidental causes". The Australian Institute of Health and Welfare quotes the maternal death ratio for Australia to be 8.4 per 100,000 of women giving birth, which equates to the WHO maternal mortality ratio (MMR) for Australia ${ }^{2,7}$. The WHO estimates MMR for developed regions to be 14 per 100,000 compared to 240 per 100,000 in developing regions of the world ${ }^{7}$. Risk factors for pregnancy associated death include: age over 35 years, women with multiple pregnancies, obese women (BMI greater than 40), late booking or nonattendees of antenatal care, those subjected to domestic violence, substance abusers and black African and Caribbean women ${ }^{8}$. 


\section{Conclusion}

Maternal collapse is a frightening and unpredictable event, but with a prepared team, adverse outcomes may be minimized. When adverse events do occur, effective communication between the various specialties, obstetricians, anaethetists and paediatrics, is essential and all team members need to be included in the debriefing process. The development of departmental guidelines with respect to maternal collapse will also help to stimulate discussion and ensure everyone is aware of causes and management of such events. Finally, it is important to remember that maternal deaths are a rare occurrence and one that may be unavoidable despite prompt attention and exceptional care.

\section{References}

1. Vanden Hoek TL, Morrison LJ, Shuster M, Donnino M, Sinz E, Lavonas EJ, Jeejeebhoy FM, Gabrielli A. Part 12: cardiac arrest in special situations: 2010 American Heart Association guidelines for cardiopulmonary resuscitation and emergency cardiovascular care. Circulation 2010; 122(suppl 3): S829-S861.

2. Royal College of Obstetricians and Gynaecologists. Green top guideline no. 56. Maternal collapse in pregnancy and the puerpurium; January 2011.

3. Sullivan EA, Hall B, King JF. Maternal deaths in Australia 2003-2005. Maternal deaths series no.3. Cat. No. PER 42. Sydney: AIWH national Perinatal Statistics Unit 2007.

4. Australian Resuscitation Council. Guideline 11.10 Resuscitation in special circumstances. In: Australian Resuscitation Council, editor. Advanced Life Support. November 2011.

5. Katz VL, Dotters DJ, Droegemueller W. Perimortem cesarean delivery. Obstet Gynecol 1986; 68: 571-76.

6. Katz VL, Balderston K, DeFreest M. Perimortem cesarean delivery: Were our assumptions correct? Am JObstet Gynecol 2005; 192: 1916-21.

7. World Health Organization. Trends in maternal mortality: 1990 to 2008. 2010.

8. Neilson JP. Maternal mortality. Obstet Gynaecol Reprod Med 2008; 19:2: 33-6. 